\title{
INVESTIGATION OF HIGH SPEED CAVITATION FLOWS
}

The paper presents an investigation of cavitating flow behind a micro-orifice. Experiments were performed with a device making visualization of a cavitation cloud possible. Two micro-orifices of the sizes $0.58 \mathrm{~mm}$ and $0.35 \mathrm{~mm}$ were tested at different cavitation numbers and at different flow rates. Parameters of the cavitation cloud collapse on a smooth surface were found. The experimental device, experiment methodology and some results are presented. Results of visualization are compared with CFD simulations.

Keywords: Micro-orifice, cavitation cloud, visualization, Lichtarowicz cell

\section{Introduction}

High speed cavitation flows when a cavitation cloud collapses due to the high flow speed far from a point of its origin occur in many hydraulic devices. Such devices usually contain sealing gaps or openings and work with high pressure gradients of liquid. The liquid flows through such gaps at relatively high velocities (tens or hundreds of $\mathrm{m} / \mathrm{s}$ ). The consequence of the increased flow speed is a local pressure drop causing a cavitation phenomenon. The cavitation cloud then collapses away in a high pressure region and if it is impinging on a solid wall a very intensive damage is possible. There are also some other applications where the erosive effect has a positive response and is intensified. We can mention cutting of materials, non-chemical etching of surfaces, peening of metal surfaces, etc. The aim of our investigation is the research of high-speed cavitation flows behind micro-orifices. Very important part of the research is a visualization of the cavitation cloud requiring special methods of illumination as well as data recording. Obtained results are compared with CFD simulations.

\section{High speed cavitation flows in micro-orifices}

Intensity of cavitation depends on the cavitation number. The cavitation number $\sigma$ for an orifice is defined as follows:

$$
\sigma=\frac{P_{2}-p_{v}}{\frac{v_{2}^{2}}{2} \rho}
$$

where $p_{2}$ - downstream pressure, $p_{v}$ - vapor pressure, $v_{2}$ - the output velocity, $\rho$ - density.

For the jet holds the Bernoulli's equation in a form (if a velocity $v_{1}$ is neglected with respect to the velocity $v_{2}$ ):

$$
\frac{p_{1}-p_{2}}{\rho}=C_{v}^{2} \frac{v_{2}^{2}}{2} \quad(\mathrm{~J} / \mathrm{kg})
$$

\footnotetext{
* Robert Olsiak, Branislav Knizat, Marek Mlkvik

Faculty of Mechanical Engineering, STU Bratislava, Slovakia, E-mail: robert.olsiak@stuba.sk
}

After combining equations (1) a (2) we obtain:

$$
\frac{1}{\sigma}=\frac{1}{C_{v}^{2}} \frac{p_{1}-p_{2}}{p_{2}-p_{v}}
$$

With respect to the fact that the velocity coefficient $C_{v}$ is constant, we obtained a linear relation between the parameter $\sigma^{-1}$ and the parameter $\frac{p_{1}-p_{2}}{p_{2}-p_{v}}$. This relation - formula (3) holds only in a cavitation regime of flow.

\section{Test rig}

A base part of the test rig is so called Lichtarowicz cell (modified in this case). The cell is designed for the research of flow of a cavitation cloud and for the research of its erosive effects. The cloud impinges on a sample placed in a fixed distance from the jet. The cell has a circular frame; the jet opening and the sample are lying in the same line. The basic schema of the experimental set up for cavitation flow visualization is presented in Fig. 1. Experimental configuration of visualization parts is integrated from the following major components and subsystems. Part No. 1 is a special hydraulic chamber. Cavitation cloud near a back side of a micro orifice is observed via visualization method. Most sensors for measurements of hydraulic parameters are installed in a cell across the construction walls. Part No. 2 is a high speed digital video camera. It is the monochromatic digital camera RedLake Y3 based on CMOS video chip with very low inter frame time (less than $100 \mathrm{~ns}$ in a double rate mode). The digital camera has an integrated 4 GB video memory and digital communication links USB 2.0 or Giga Ethernet. Part No. 3 is DAQ, a vision and control system. The system is integrated at the Windows XP Workstation platform. DAQ and a control $\mathrm{I} / \mathrm{O}$ card used in this system for functionality expansion are from the National Instruments product range. The DAQ I/O card is used for measurements of hydraulic parameters: inlet pressure, pressure in a test chamber, test liquid temperature, 
mass flow, etc. Software Motion Studio (Motion Studio Pro for selected analysis) is used for vision applications. Part No. 4 is the NANOLITE flash lamp with a very low flash duration, typically $8 \mathrm{~ns}$. The lamp is a source of a cold white light with an extreme intensity. Part No. 5 is the MINISTROBOKIN flash driver which is used with the NANOLITE flash lamp. The lamp is a high repetition (up to $20 \mathrm{kHz}$ ) high voltage pulse generator, synchronized and triggered via analog TTL technology. The operation mode is controlled by the DAQ system. A subsystem No. 6 is a pressurized air circuit. This circuit is applied for blowing the plasma torch incipient at NANOLITE flash lamp electrodes. However, it is a standard pneumatic system, but the application is unconventional. The test rig is also described in [1]

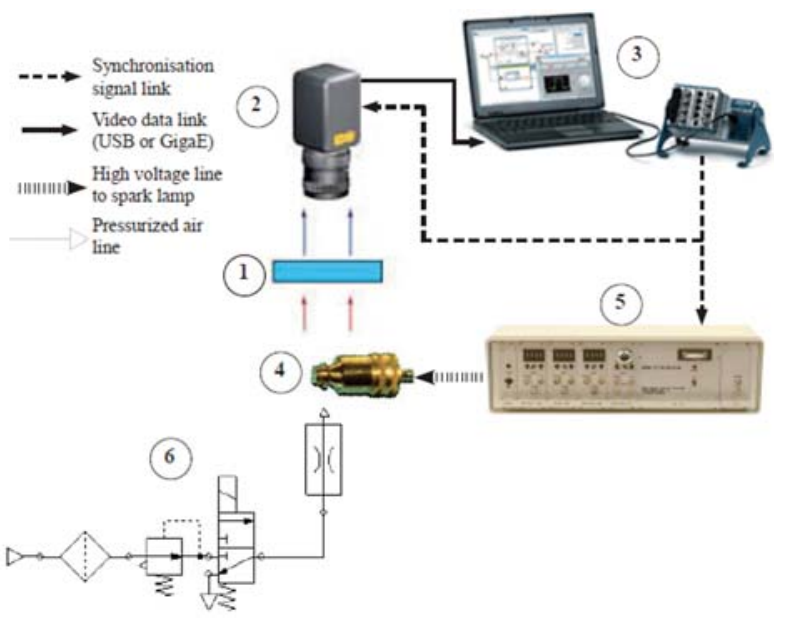

Fig. 1 Schema of the experimental test rig

\subsection{Video recording and illumination}

Acquired data from the described experimental system have two marginal characteristics: numerical data - acquired values from analog physical signals (e.g. pressure, temperature, mass flow) and calculated values (e. g. cavitation number) which are stored as a data file. Video data from the experiments are stored in the digital camera video memory 2 in a first step, in a second step the data are transmitted to the Vision software via communication link (USB 2.0 as standard) and stored as a digital video file (HDD of DAQ and Vision system) 3. The format of the video file is optional, but saving as *.RAW format is necessary for an advanced post-analysis. Via decoding the stored *.RAW video file the set of the digital pictures is available for a detailed analysis and advanced measurements, Fig. 2.

In Fig. 2 the region of interest (ROI) is defined - a space between the tested orifice back side and a face side of the specimen (see flow direction in Fig. 2, too). The intensity of light and shape contours of solid bodies in the defined region is strictly required for video data analysis, especially for a quantitative analysis algo-

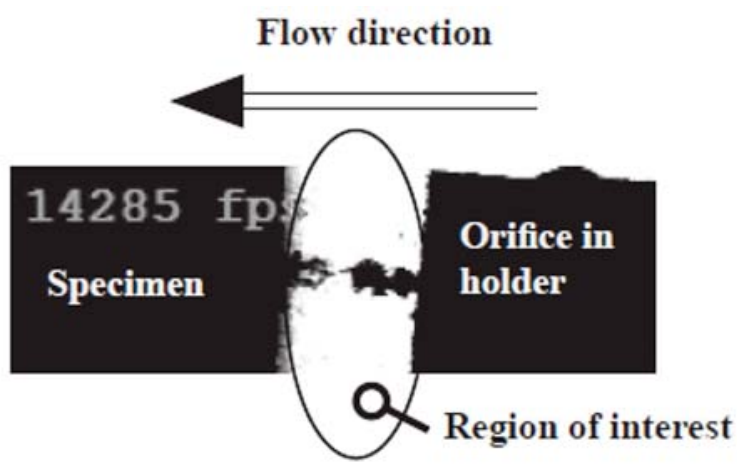

Fig. 2 Sample image isolated from experimental video sequence acquired@14285 framesper second

rithm. The shape of contours of the solid bodies in the ROI is dependent on the used optical component and the vision system settings. Lighting conditions are strictly dependent on the quality and stability of the illuminate apparatus (light source). The flash lamp is used as the illuminating equipment. The flash lamp is improved up to the optimum efficiency offering the shortest possible flash duration. Due to the extremely high luminous density of the point shaped spark the lamp has a sufficiently high flash energy. Some other applications areas of the nanosecond flash illumination for the photographic analysis of extremely fast events are, e.g., in shock wave tubes, hypersonic wind tunnels, droplet research (PIV), bubble formation, optical stress experiments, shadow and interferometer photography. The simple spark is produced by the instantaneous discharge of electric energy, stored in a capacitor, through a system of HVE and GE electrodes Fig. 3. The most important characteristics of the flash light source are the light energy, flash duration, form of the luminous spark plasma, and possibly its spectral composition. The latter is mainly determined by the atmosphere in which the discharge takes place. A simple air spark consists of almost white light. For the most applications of flow visualization it is desirable to have a spark volume as small as possible, e.g., a point-shaped light source. In the arrangement in Fig. 3, the luminous spot appears small to an observer in the direction of the object field, since the spark is forced to discharge through the ceramic capillary tube. The flash time is determined by the inductivity of the discharge circuit; this must be kept as low as possible. The illumination system is described in detail for example in [2].

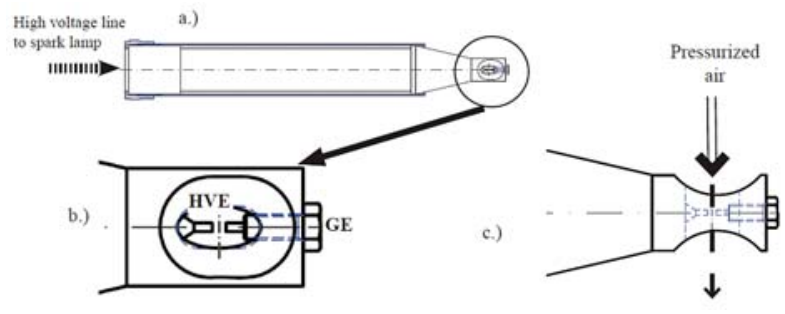

Fig. 3 a) Construction of high voltage spark lamp. b) The high voltage and ground electrodes. c) Stabilization of plasma properties by the pressurized air 


\subsection{Micro-orifices}

The experiment was carried out with two different micro-orifices $d=0.35$ a $d=0.58 \mathrm{~mm}$.

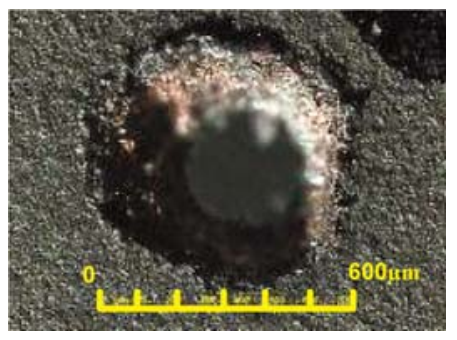

$a$

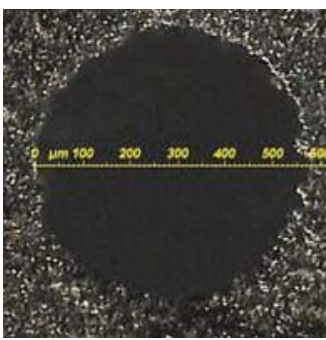

$b$
Fig. 4 Micro-orifice $0.350 \mathrm{~mm}$ (left) and $0.580 \mathrm{~mm}$ (right)

In Fig. 4 is a photo of both $0.35 \mathrm{~mm}$ and $0.580 \mathrm{~mm}$ micro-orifices. Micro-orifices were fixed in the holder by sticking. The material of the micro-orifices is hard alloy and they were manufactured in UTM SjF STU in Bratislava.

\section{Numerical simulation of cavitating flows}

\subsection{Computational grid}

Computational domain for the simulation is created as 2-Dimensional and axissymetric. This simplification allows us to use square grid elements. This approach allows us strongly reduce the computation time. Also the quality the grid elements is much more better as in the 3-Dimensional version of the grid, which produces the elements, where the high skew of the elements is often a problem. The length of the element's corner is set to $1 / 10$ of the orifice diameter. This size is set according to our previous experiences with the simulation of the cavitating flows.

The boundary conditions are set according to Fig. 5 .

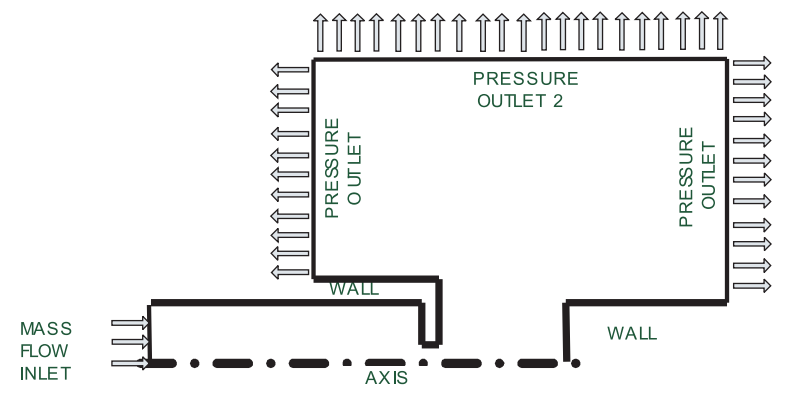

Fig. 5 Used 2-D axisymmetric computational domain with boundary types

\subsection{Solution setup}

The solution of the cavitation jet is set as a transient one. According to the very high characteristic speed of the cavitation jets, the time step of the solution is set $10^{-5} \mathrm{~s}$. Very important for a CFD simulation is to set correct material properties. In this case, the properties of air and water vapor must be assumed as compressible. Other simulations parameters can be found in Tab. 1 . As can be seen in [3], the well tuned numerical model can successfully solve a problem of the cavitating flow.

Solution Setup

Tab. 1

\begin{tabular}{|l|l|}
\hline $\begin{array}{l}\text { Computational Domain and } \\
\text { The Grid }\end{array}$ & $\begin{array}{l}\text { 2D, axisymetric, structural grid(quads), } \\
\text { element length scale is } 10 \% \text { of the orifice } \\
\text { radius }\end{array}$ \\
\hline Solution Type & Transient solution (time step $\left.\sim 10^{-5} \mathrm{~s}\right)$ \\
\hline Model of Turbulence & $k-\varepsilon$ \\
\hline Solver type & SIMPLE \\
\hline Multiphase model & Mixture, Eulerian \\
\hline $\begin{array}{l}\text { Model of the phase change } \\
\text { (cavitation model) }\end{array}$ & Singhal Model, Model Zwart-Gerber \\
\hline Liquid phase properties & $\begin{array}{l}\text { incompressible, properties according to } \\
\text { the normal conditions }\left(20^{\circ} \mathrm{C}, 1 \text { bar) }\right.\end{array}$ \\
\hline Gases properties & $\begin{array}{l}\text { Compressible- state equation of the ideal } \\
\text { gas, }\end{array}$ \\
\hline
\end{tabular}

\section{Results of investigation}

\subsection{Results of visualisation}

The experiments were carried out with two micro-orifices in a wide range of flow rates, pressures and cavitation numbers. In Fig. 6 is a typical structure of a cavitating cloud impinging on a solid wall.

As it is depicted in Fig. 6, the cloud flows from the opening of a diameter $\mathrm{d}$ and impinges on a sample at a distance $x$. The cloud collapses on the sample and the region of collapse is denoted $D$. The structure of the cloud is not stable; it is periodically changing,

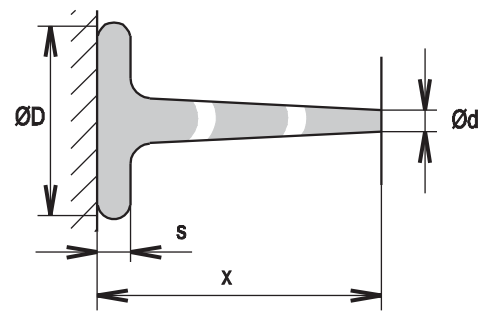

Fig. 6 Structure of a jet impinging on a solid wall 

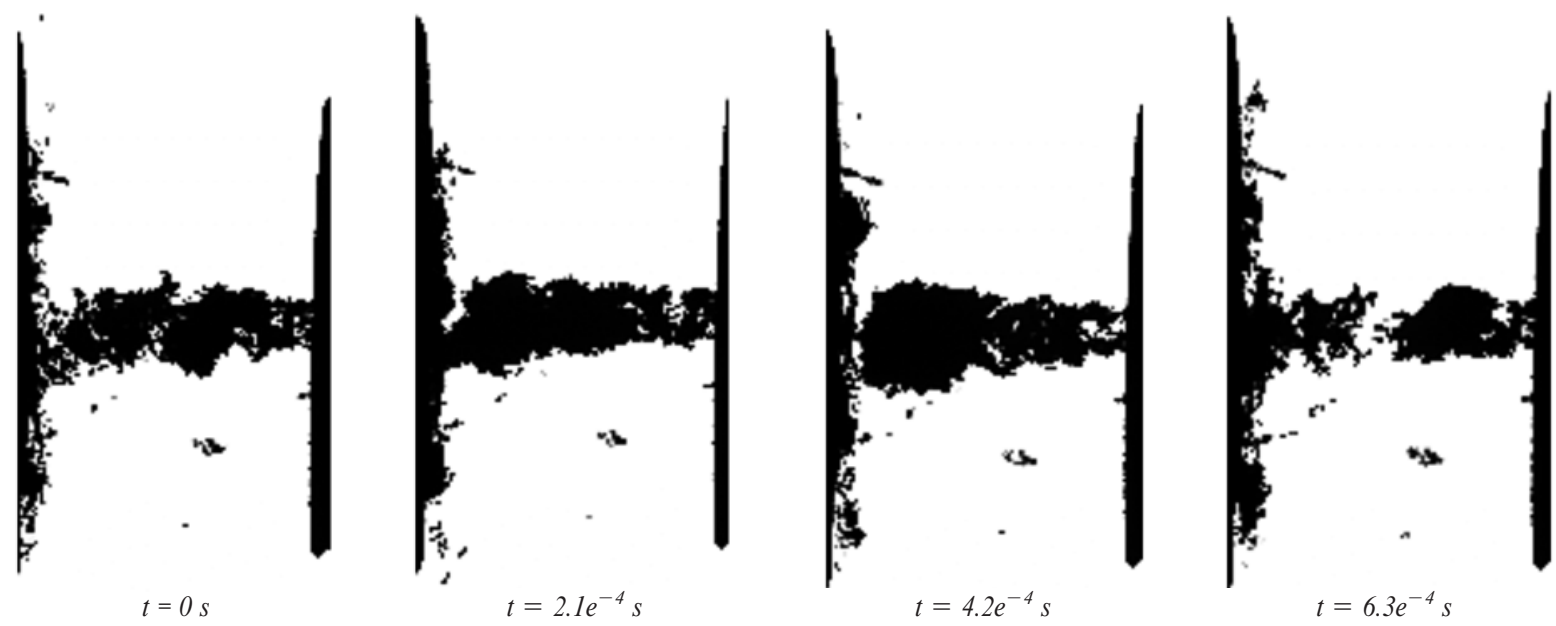

Fig. 7 Visualization of flow. Orifice diameter $0.58 \mathrm{~mm}$, pressure $p_{1}=17 \mathrm{bar}$

which is noticeable especially at higher values of a cavitation number. In Fig. 7 is depicted a sequence of shots of flow for the orifice with a diameter $d=0.580 \mathrm{~mm}$.

From the figure it is clear that the stream is periodically changing. In Fig. 8 is depicted a typical shape and structure of the jet in dependence on the cavitation number. An opening with a diameter $d=0.580 \mathrm{~mm}$ and a distance $x=5.0 \mathrm{~mm}$ between the jet and the sample is selected. At high cavitation numbers the cloud collapses even before it reaches the sample. With the decreasing of $\sigma$ the intensity of a cloud grows. At the same time the diameter $D$ (Fig. 6) of the region on which the cloud collapses is increasing.

In Fig. 9 are depicted nondimensional geometrical parameters of a cavitating flow for both micro-orifices. It is a nondimensional cavitation cloud thickness $s / d$ and a nondimensional diameter of a collapse $D / d$. The curves are presented in dependence on the cavitation number $\sigma$. With a decreased value of $\sigma$ the intensity of cavitation grows and the thickness and diameter of a collapsing cloud also grow. The distance between the jet and sample has also an effect. For example, at a distance $x=5.0 \mathrm{~mm}$ at the greatest values of cavitation number $(\sigma=0.28)$ the cloud doesn't reach the sample. That is the reason why the cavitation effects (especially

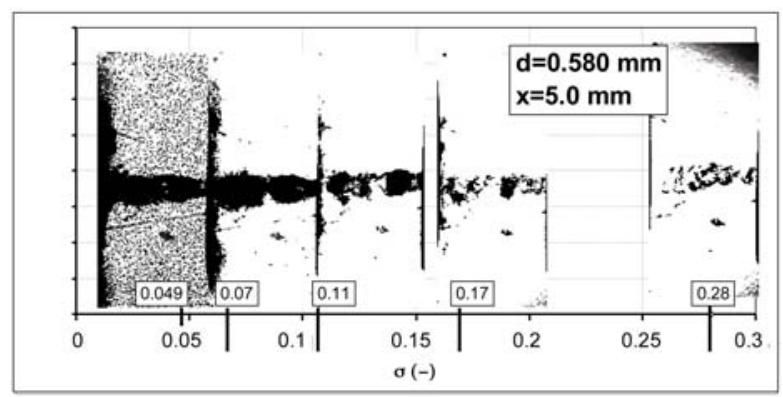

Fig. 8 Structure of a jet in dependence on the cavitation number erosion) are strongly dependent on the cavitation number. The experiments show that the magnitude of this number influences the region of a cavitation cloud collapse.
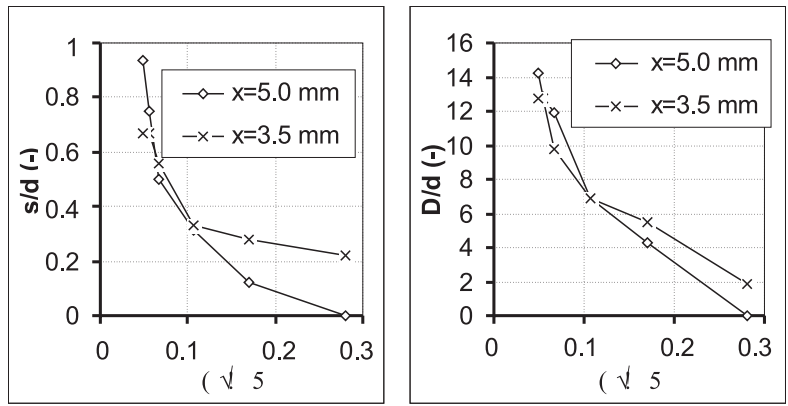

Fig. 9 Relative thickness (left) and relative diameter (right) of a cavitation cloud for the orifice $0.580 \mathrm{~mm}$

\subsection{Comparison of experiments and CFD calculations}

As shown in Fig. 10, both of the used cavitation models are able to compute the pressure loss with a good accuracy. It can be observed that the computed inlet pressure is in both cases higher than the inlet pressure measured. The results obtained with the Singhal cavitation model are more accurate, especially in the case of higher cavitation numbers. The Singhal cavitation model is then more useful in the case of flow where the cavitation process is in its early stages (cavitation inception).

The visualized structure of the cavitation cloud can be compared with simulation results. Computed distribution of the vapor phase can be seen in Figs. 11 and 12. Both of the cavitation models give results which are comparable with the experimental data. Again, the Singhal cavitation model is more accurate even in higher cavitation numbers. 


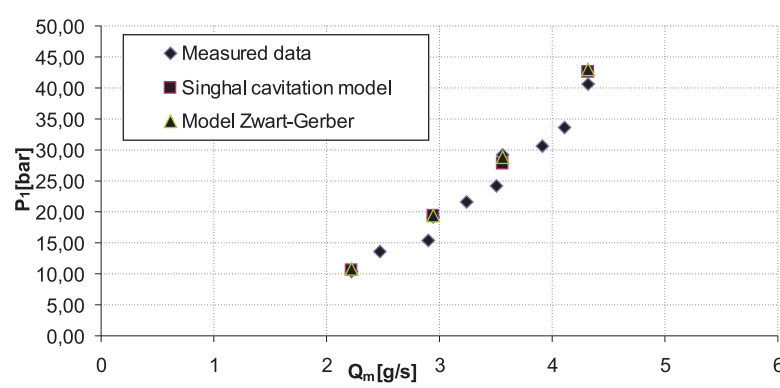

Fig. 10 Comparison of the pressure loss in the orifice (the simulation and the experiment)

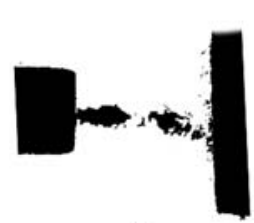

a

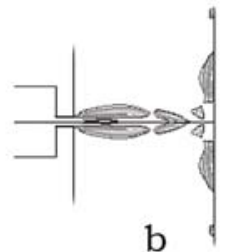

b

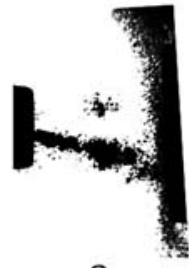

a

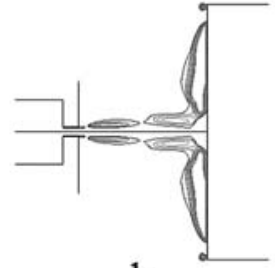

b

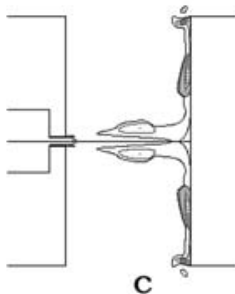

C
Fig. 12 Comparison of the spatial distribution of vapor for $\sigma=0.32$ ( $a$ - experiment, $b$ - Singhal cavitation model, $c$ - Zwart-Gerber cavitation model)

\section{Conclusion}

In the paper the visualizations of flow of a cavitation cloud behind the micro-orifice are presented. The shots of a collapsing cloud on a sample for two micro-orifices of different sizes are also presented. Generally it may be stated that the intensity of the cloud which is in contact with the sample depends on a cavitation number, micro-orifice size, and distance. During the experiments speeds up to $100 \mathrm{~m} / \mathrm{s}$ were reached behind the micro-orifice. This fact requests special approach to images acquisition and primarily to the illumination of the cavitating micro-jet. Obtained results of visualization are compared with the results of CFD simulations. A good agreement between simulations and visualizations can be seen from the comparisons. However, it is necessary to choose a suitable cavitation model in dependence on the magnitude of the cavitation number. Also the quality of the computational grid has strong effect on the simulation results. In presented case, the square elements were used to generate the 2-D axissymetric grid.

\section{Acknowledgement}

This work was supported by the Scientific Grant Agency VEGA under contract number 1/0215/11.

\section{References}

[1] OLSIAK, R., KNIZAT, B., MLKVIK, M.: Visualization of Cavitating Micro Jets. Experimental Fluid Mechanics 2011, Proc. of the Intern. Conference, Jicin, 2011.

[2] MLKVIK, M., OLSIAK, R., KNIZAT, B.: Vizualization of the Cavitating Jet Using Flashlamp For Illumination. Experimental Fluid Mechanics 2011, Proc. of the Intern. Conference, Jicin, 2011.

[3] KOZUBKOVA, M.: Matematicke modely kavitace a hydraulickeho razu [Mathematical Models of Cavitation and Hydraulic Impact]. VSB- Technical University Ostrava, 2009, ISBN 978-80-248-2043. 